\title{
Random assignment and shortest path problems
}

\author{
Johan Wästlund If $^{\Uparrow}$ \\ ${ }^{1}$ Department of Mathematics, Linköping University, 58183 Linköping, Sweden
}

We explore a similarity between the $n$ by $n$ random assignment problem and the random shortest path problem on the complete graph on $n+1$ vertices. This similarity is a consequence of the proof of the Parisi formula for the assignment problem given by C. Nair, B. Prabhakar and M. Sharma in 2003.

We give direct proofs of the analogs for the shortest path problem of some results established by $\mathrm{D}$. Aldous in connection with his $\zeta(2)$ limit theorem for the assignment problem.

Keywords: minimum matching, random tree

\section{Introduction}

The purpose of this article is to bring together some results on random assignment and shortest path problems, and to clarify how they relate to each other. In particular, we investigate the consequences of the two very different and hard proofs of the $\zeta(2)$ limit theorem for the random assignment problem given by David Aldous [1] and by Chandra Nair, Balaji Prabhakar and Mayank Sharma [11].

The random shortest path problem had already been investigated by Svante Janson [6] (and has recently been further studied by R. Van der Hofstad, G. Hooghiemstra and P. Van Mieghem [4; 5]). By putting the papers [11] and [6] side by side, one can see that there is a close connection between these two random optimization problems.

By establishing this connection we show that some results of Aldous [1] must hold also for the shortest path problem. Actually these properties are easier to establish directly for the shortest path problem, and by doing so we obtain, via the results of [11], new independent proofs of Aldous' results.

\section{Random assignment problems}

The $n$ by $n \exp (1)$ assignment problem can be defined as follows: The entries of an $n$ by $n$ matrix are independent $\exp (1)$ random variables. An assignment is a set of $n$ matrix positions, such that each row and column contains exactly one of them. The cost of the assignment is the sum of these matrix entries.

A random variable that has been studied for a long time is the minimum cost $C_{n}$ of an assignment. In 1979 D. Walkup [14] showed that $E\left(C_{n}\right)$ remains bounded as $n \rightarrow \infty$. It was conjectured by Marc Mézard and Giorgio Parisi [8, 9; 10] that as $n \rightarrow \infty$,

$$
E\left(C_{n}\right) \rightarrow \frac{\pi^{2}}{6}
$$

In her PhD thesis [12] in 1992, Birgitta Olin gave further evidence for the Mézard-Parisi conjecture by simulation, and proved an asymptotic lower bound of 1.51 . From her simulations, she made the interesting observation that in about half of the rows of the matrix, the smallest entry in the row is also the one that participates in the minimum assignment. She investigated this further, and discovered what we will call the $2^{-i}-l a w$ :

Theorem 1 Let the ordered sequence of entries in a particular row of the matrix be $x_{1} \leq x_{2} \leq \cdots \leq x_{n}$. Then for each positive integer $i$, the probability that $x_{i}$ participates in the minimum assignment converges to $2^{-i}$ as $n \rightarrow \infty$.

In 2001, the limit [1] was proved by David Aldous [1]. In the same paper, he also gave a proof of Theorem 1

\footnotetext{
†jowas@mai.liu.se
} 


\section{Exact formulas}

In 1998, Parisi suggested another way of proving [1]. He conjectured in [13] that for every $n, E\left(C_{n}\right)$ is given by the exact formula

$$
E\left(C_{n}\right)=1+\frac{1}{4}+\frac{1}{9}+\cdots+\frac{1}{n^{2}}
$$

This conjecture was verified simultaneously and independently in 2003 by Chandra Nair, Balaji Prabhakar, and Mayank Sharma [11], and by Svante Linusson and the author [7]. The Linusson-Wästlund proof gives, as a by-product, an exact formula for the $i=1$ case of the $2^{-i}$ law. It is proved in [7] that the probability that the smallest entry in a row participates in the minimum assignment is

$$
\frac{1}{2}+\frac{1}{2 n}
$$

Naturally one can ask whether there are similar exact formulas for $i \geq 2$, but the approach taken in [7] does not seem to give any information about this. We show that from the proof of (2) given by Nair, Prabhakar and Sharma, one can deduce not only the formula (3), but also exact results for the higher values of $i$, allowing a new proof of Theorem 1 . In particular, we show that the probability that the second smallest entry in a row participates in the minimum assignment is given by the formula (4) below.

It is quite remarkable that although the three proofs of $(1)$ given in $[1,7 ;, 11]$ are very different, they all give the $i=1$ case of Theorem 1 as a by-product, and two of them allow proofs of the full theorem.

\section{The shortest path in a random graph}

It is tempting to try to find a simple explanation of the formula 3 . It seems as if with probability $1 / n$, the smallest entry will participate "just by chance", and if this is not the case, then with probability exactly $1 / 2$, it will participate anyway because it is smallest.

There is another type of random minimization problem where precisely this situation occurs. This is the problem of the minimum length path between two vertices in the complete graph, where the edge lengths are chosen independently from $\exp (1)$-distribution.

We consider this problem on the complete graph $K_{n+1}$ on $n+1$ vertices $v_{0}, \ldots, v_{n}$. We choose two vertices $v_{0}$ and $v_{n}$ and ask for the probability that the shortest edge from $v_{0}$ belongs to the shortest path from $v_{0}$ to $v_{n}$. If "by chance" the shortest edge from $v_{0}$ happens to go directly to $v_{n}$, then this is of course also the shortest path to $v_{n}$. Suppose therefore that the shortest edge from $v_{0}$ goes to another vertex, say $v_{1}$. Then we can subtract the length of the edge $\left(v_{0}, v_{1}\right)$ from the lengths of all edges from $v_{0}$, including the one to $v_{1}$. In this new problem, the edge $\left(v_{0}, v_{1}\right)$ has zero length, but all other edge lengths are still independent and $\exp (1)$-distributed. Since every path from $v_{0}$ to $v_{n}$ contains exactly one edge from $v_{0}$, the same amount has been subtracted from all of them, so the shortest path will still be the same. Moreover, it is clear by symmetry that the probability that the edge $\left(v_{0}, v_{1}\right)$ belongs to the shortest path from $v_{0}$ to $v_{n}$ is exactly $1 / 2$, since this happens if and only if without using the edge $\left(v_{0}, v_{1}\right)$, the distance from $v_{1}$ to $v_{n}$ is shorter than the distance from $v_{0}$ to $v_{n}$. It follows that the participation probability of the shortest edge from $v_{0}$ is given by $(3)$.

This leads to the question whether the participation probability of the $i$ :th shortest edge for $i \geq 2$ is also the same in the two problems. We show that this is the case. Moreover, we give an independent proof that the $2^{-i}$-law holds also for the shortest path problem, thereby obtaining a new proof of Theorem 1 .

\section{Combining the results on shortest paths and minimum assign- ments}

The shortest path problem was studied in detail by Svante Janson [6] in 1999 and more recently by R. Van der Hofstad, G. Hooghiemstra and P. Van Mieghem [4, 5]. We first give a short account of some of Janson's results, and now we consider the complete graph $K_{n}$ on $n$ vertices.

Janson used the first passage percolation model, where an infection starts at a root vertex, which we now take to be $v_{n}$, and spreads through each edge with $\exp (1)$ rate. It is easily observed that the length of the minimum path from $v_{n}$ to another vertex $v_{j}$ is equal to the time at which $v_{j}$ is infected. If, at a certain moment, $k$ vertices are infected, then the time until the next vertex is infected is $\exp (k(n-k))$-distributed, since there are $k(n-k)$ edges that connect an infected vertex to a non-infected one. Moreover, these waiting times are independent. 
Let $t_{1}, \ldots, t_{n}$ be the ordered sequence of distances from the vertex $v_{n}$ to all the vertices of the graph, including $v_{n}$ itself. Equivalently, $t_{1}, \ldots, t_{n}$ are the times at which a new vertex gets infected. Then $t_{1}=0$, and the increments $t_{k+1}-t_{k}$ are independent, with $t_{k+1}-t_{k} \sim \exp (k(n-k))$.

In [11], the proof of the Parisi conjecture [2] is based on a result on minimum assignments in certain submatrices, with a remarkable similarity to Janson's results on minimum paths. In an $n-1$ by $n$ matrix of independent $\exp (1)$ entries, consider the costs of the minimum assignments in the $n$ submatrices obtained by deleting one of the columns. Let $T_{1}, \ldots, T_{n}$ be the ordered sequence of these costs. The following theorem was proved by Nair, Prabhakar and Sharma [11]:

Theorem 2 The increments $T_{k+1}-T_{k}$ are independent, and $T_{k+1}-T_{k} \sim \exp (k(n-k))$.

This is a quite deep theorem, and as we explain in Section 9, it is relatively simple to prove that the Parisi formula (2) follows from it. It has the consequence that we can couple the random $n-1$ by $n$ matrix with the edge lengths in $K_{n}$ in such a way that $T_{k}=T_{1}+t_{k}$ for every $k$. Suppose now that we introduce another vertex $v_{0}$ with independent $\exp (1)$ edge lengths $x_{1}, \ldots, x_{n}$ to the $n$ vertices $v_{1}, \ldots, v_{n}$, and that we extend the $n-1$ by $n$ matrix by another row with independent $\exp (1)$ entries $X_{1}, \ldots, X_{n}$. Then we can couple the $n$ by $n$ matrix with the edge lengths in $K_{n+1}$ in such a way that $X_{k}=x_{k}$ for every $k$, and the entry $X_{k}$ participates in the minimum assignment if and only if the edge of length $x_{k}$ participates in the minimum path from $v_{0}$ to $v_{n}$. This is because the minimum assignment in the matrix consists of an entry $X_{i}$ together with the minimum assignment in the matrix obtained by deleting column $i$ in the original $n-1$ by $n$ matrix, and similarly the shortest path from $v_{0}$ to $v_{n}$ in the complete graph consists of an edge $\left(v_{0}, v_{i}\right)$ (here possibly $i=n$ ) together with the shortest path from $v_{i}$ to $v_{n}$.

\section{Proof of the $2^{-i}$-law for the shortest path problem}

In the percolation model, we now let the infection start at the vertex $v_{0}$, and spread to the vertices $v_{1}, \ldots, v_{n}$ by rate 1 through every edge. If we do not care about the actual times that the infection spreads, we can think of this as a process in discrete time, where at each step, an edge is chosen uniformly among all edges that connect an infected vertex to a non-infected one. The infection spreads through this edge, and the edges that are chosen in this way form a tree that grows from $v_{0}$ and eventually reaches all vertices.

Instead of fixing another vertex, say $v_{n}$, and asking for the probability that the $i$ :th shortest edge from $v_{0}$ belongs to the shortest path from $v_{0}$ to $v_{n}$, we ask for the expected number of vertices among $v_{1}, \ldots, v_{n}$ whose shortest path from $v_{0}$ starts with the $i$ :th shortest edge. This is of course the same thing apart from a factor $n$. The following lemma shows that it suffices to show that the $2^{-i}$-law holds in the discrete percolation model.

Lemma 1 If we fix $i$ and let $n \rightarrow \infty$, then with high probability, the $i$ :th edge grown from the root $v_{0}$ in the percolation model is also the $i$ :th shortest edge from $v_{0}$.

Proof: We have to show that with high probability, the $i$ shortest edges from the root are also the shortest paths to the vertices they go to. This follows easily from the results of Janson [6]. The typical distance between two randomly chosen vertices in the complete graph is about $\log n / n$, and moreover the probability that the distance is smaller than, say, $\log n /(2 n)$ tends to zero as $n \rightarrow \infty$. Therefore with high probability in the limit $n \rightarrow \infty$, the $i$ shortest edges from the root are all of length smaller than $\log n /(2 n)$, and for none of them there is a shortcut, that is, some other path of smaller length to the same vertex.

We can now give a proof of the $2^{-i}$-law for the shortest path problem. By the coupling described in the previous section, this gives a new proof of Theorem 1 .

Theorem 3 Let the edges of the complete graph on the vertices $v_{0}, \ldots, v_{n}$ have independent $\exp (1)$ lengths. Let the ordered sequence of edge lengths from the vertex $v_{0}$ be $x_{1} \leq x_{2} \leq \cdots \leq x_{n}$. Then for each positive integer $i$, the probability that the edge of length $x_{i}$ participates in the shortest path from $v_{0}$ to $v_{n}$ converges to $2^{-i}$ as $n \rightarrow \infty$.

Proof: We let $m_{i}$ be the number of vertices that are reached from the root through the $i$ :th edge grown from the root in the percolation model (and $m_{i}=0$ if there are fewer than $i$ such edges). By symmetry,

$$
E\left(m_{1}\right)=\frac{n+1}{2}
$$


since the root and the vertex reached in the first step of the percolation are competing on equal terms for the remaining vertices. Similarly, for $i \geq 2$, if $k$ is the number of vertices that are not reached through any of the first $i-1$ edges from the root, then conditioning on $k>0$ we have

$$
E\left(m_{i}\right)=\frac{k+1}{2},
$$

while obviously $m_{i}=0$ if $k=0$. By induction on $i$ it follows that for fixed $i$, as $n \rightarrow \infty$, we have

$$
E\left(m_{i}\right) \frac{n}{2^{i}}+O(1)
$$

where the error term comes from the probability that $m_{j}=0$ for some $j<i$. Therefore the probability that a given vertex, say $v_{n}$, is reached through the $i$ :th edge from the root is

$$
2^{-i}+O(1 / n)
$$

In view of Lemma 1] this completes the proof.

\section{An exact formula for the second shortest edge}

We prove an exact formula for the probability that the second smallest matrix entry in a row participates in the minimum assignment, or equivalently, that the second shortest edge from the root participates in the shortest path to a particular vertex. However, since this formula turns out to be quite complicated, our conclusion is that the formula is in itself of limited interest.

We consider the percolation process described earlier, and condition on the number $i$ of vertices apart from the root that are infected before the second edge is grown from the root. The probability that this number is equal to $i$ is given by

$$
\frac{1}{i(i+1)} \text {. }
$$

Given that this happens, the vertex connected to the root by the second edge from the root will compete for the remaining vertices on equal terms among the $i+2$ vertices that are infected at this stage. Hence the expected number of vertices that are reached through the second edge from the root is

$$
1+\frac{n-i-1}{i+2}
$$

It remains to find the probability that the second edge grown from the root in the percolation process is also the second shortest edge from the root. Let $Y_{i}=t_{i+1}-t_{i}$ be the times between the infections of new vertices. The first edge grown from the root has length $Y_{1}$. Then $i-1$ more vertices are infected through this edge, and the lengths of the edges from the root to these vertices, say $v_{2}, \ldots, v_{i}$, are $Y_{1}+Y_{2}+Z_{2}, Y_{1}+$ $Y_{2}+Y_{3}+Z_{3}, \ldots, Y_{1}+\cdots+Y_{i}+Z_{i}$, where $Z_{1}, \ldots, Z_{i}$ are $\exp (1)$ variables, independent of everything else. The next edge to spread the infection is the second edge from the root, which has length $Y_{1}+\cdots+Y_{i+1}$.

In order for this edge to be the second shortest of the edges from the root, first of all $Y_{i+1}$ must be the smallest of the numbers $Z_{2}, \ldots, Z_{i}, Y_{i+1}$. Conditioning on this is equivalent to conditioning on the event $Y_{i+1}=0$. Given this, we can exclude the edge of length $Y_{1}+\cdots+Y_{i}+Z_{i}$, and then $Y_{i}$ must be smallest of the numbers $Z_{2}, \ldots, Z_{i-1}, Y_{i}$ and so on. Since $Y_{i}$ is exponential of rate $i(n-i+1)$, the probability for all this is

$$
\prod_{j=1}^{i-1} \frac{(i+2-j)(n-i-1+j)}{i-j+(i+2-j)(n-i-1+j)} .
$$

Consequently, the probability that the second shortest edge from $v_{0}$ belongs to the shortest path from $v_{0}$ to $v_{n}$ is given by

$$
\frac{1}{n} \sum_{i=1}^{n-1} \frac{1}{i(i+1)}\left(1+\frac{n-i-1}{i+2}\right) \prod_{j=1}^{i-1} \frac{(i+2-j)(n-i-1+j)}{i-j+(i+2-j)(n-i-1+j)} .
$$

For $i=2,3,4,5$ this evaluates to $1 / 4,19 / 72,89 / 336$ and 2639/10000. The formula (4) can possibly be simplified slightly, but it seems more interesting to try to obtain a better understanding of the connection between the assignment and the shortest path problem in this probabilistic model. 


\section{Aldous' function $h(x)$}

The proof of (1) given by Aldous in [1] is based on the solution of the recursive distributional equation

$$
X \min \left(\xi_{i}-X_{i}\right)
$$

where $\xi_{i}$ are the times of the events in a rate 1 Poisson process, and $X_{i}$ are independent variables of the same distribution as $X$. It is proved that the logistic distribution given by

$$
P(X<x) \frac{1}{1+e^{-x}}
$$

is the unique solution to this equation. Then (1) is deduced from the following theorem:

Theorem 4 Let $x$ be a positive real number. When $n \rightarrow \infty$, the probability that a matrix entry participates in the minimum assignment given that it is equal to $x / n$, converges to a certain function $h(x)$ which is given by

$$
h(x)=P\left(x<X_{1}+X_{2}\right),
$$

where $X_{1}$ and $X_{2}$ are independent random variables of logistic distribution.

Moreover, it is shown that

$$
h(x) \frac{e^{-x}\left(e^{-x}-1+x\right)}{\left(1-e^{-x}\right)^{2}} .
$$

Theorem 4 seems to be out of reach for the method employed in [7], but again we give a new and independent proof by showing that the same result holds for the shortest path problem.

It follows from Janson's results [6] that the shortest path from $v_{0}$ to another vertex $v_{i}$ in the complete graph on $n+1$ vertices $v_{0}, \ldots, v_{n}$ is $t=Y_{1}+\cdots+Y_{k}$, where $Y_{1}, \ldots, Y_{n}$ are independent and for every $i$, $Y_{i}$ is exponential of rate $i(n+1-i)$, and $k$ is chosen uniformly on $1, \ldots, n$ independently of $Y_{1}, \ldots, Y_{n}$. If $v_{j}$ is another vertex, then the difference $\Delta t$ in distance to $v_{i}$ and $v_{j}$ is a sum $Y_{k+1}+\cdots+Y_{l}$, where $k$ and $l$ are chosen uniformly and (if we allow $i=j$ to hold with probability $1 / n$ ) independently on $1, \ldots, n$. Here the sum should be interpreted in the obvious way as a negative sum if $k>l$.

At this point we allow ourselves to be a little imprecise and just say that unless $k$ or $l$ is very close to 1 or $n$, the sum of the deviations of the variables $Y_{k+1}, \ldots, Y_{l}$ from their means will most likely be small compared to the impact that the choices of $k$ and $l$ have on the value of $\Delta t$. To find the suitably normalized limit distribution of $\Delta t$ we may therefore replace the variables $Y_{1}, \ldots, Y_{n}$ by their mean values. Hence

$$
\Delta t \approx \frac{1}{(k+1) \cdot(n-k)}+\cdots+\frac{1}{l \cdot(n+1-l)} .
$$

We can approximate $n \cdot \Delta t$ by the integral

$$
\int_{\alpha}^{\beta} \frac{d x}{x(1-x)}
$$

where $\alpha$ and $\beta$ correspond to the limits $k$ and $l$ of summation, and are therefore independent and uniform on the interval $[0,1]$. This integral can be written

$$
\int_{1 / 2}^{\beta} \frac{d x}{x(1-x)}-\int_{1 / 2}^{\alpha} \frac{d x}{x(1-x)} .
$$

It is therefore the difference of two independent equally distributed random variables whose distribution can be found as follows:

$$
\int_{1 / 2}^{\alpha} \frac{d x}{x(1-x)}=\int_{1 / 2}^{\alpha}\left(\frac{1}{x}+\frac{1}{1-x}\right) d x=\log \alpha-\log (1-\alpha) .
$$

Moreover,

$$
P(\log \alpha-\log (1-\alpha)<x)=P\left(\frac{\alpha}{1-\alpha}<e^{x}\right) P\left(\alpha<\frac{e^{x}}{1+e^{x}}\right)=\frac{e^{x}}{1+e^{x}} \frac{1}{1+e^{-x}} .
$$

Hence $n \cdot \Delta t$ converges in distribution to the difference of two independent logistic variables. Since the logistic distribution is symmetric, this is the same thing as the sum of two independent logistic variables. 
Notice however that $n(t-E(t))$ does not converge to a logistic variable, since there will be a considerable impact on $n t$ coming from the fluctuations in $Y_{i}$ for small $i$. It is only the difference in distance that converges to the difference of two independent logistic variables.

Now we can prove the analog of Theorem 4 for the shortest path problem. Let $v_{i}$ and $v_{j}$ be two arbitrary vertices in the complete graph on $n+1$ vertices $v_{0}, \ldots, v_{n}$. Consider the shortest paths from these two vertices to the vertex $v_{0}$. A priori, it is highly unlikely that the edge connecting $v_{i}$ to $v_{j}$ will belong to any of them, since this single edge will normally have length much greater than $\log n / n$, the expected distance to $v_{0}$. If we now fix a positive real number $x$, and condition on the edge $\left(v_{i}, v_{j}\right)$ having length $x / n$, then the probability that it belongs to the shortest path connecting $v_{i}$ to $v_{0}$ is the same as the probability that $x / n<\Delta t$, in other words that $x<n \cdot \Delta t$. As $n \rightarrow \infty$, this converges to Aldous' function $h(x)$.

The limit (1) can now be established as in [1] by computing the integral

$$
\int_{0}^{\infty} x \cdot h(x) d x \frac{\pi^{2}}{6},
$$

or as in [11] by first establishing the Parisi formula [2].

\section{The Parisi formula}

We can derive the Parisi formula (2) by showing that the expected length of the first edge in the shortest path between two given vertices in $K_{n+1}$ is

$$
\frac{1}{n}\left(1+\frac{1}{4}+\frac{1}{9}+\cdots+\frac{1}{n^{2}}\right) .
$$

Since we know that this is the same thing as the expected cost of the entry in the first row in the minimum assignment, (2) follows. We here point out that this follows from some recent results of R. Van der Hofstad, G. Hooghiemstra and P. Van Mieghem [4, 5]. These authors studied the shortest path tree (SPT) in the complete graph with independent $\exp (1)$ edge lengths. The SPT is the tree formed by all edges that belong to the shortest path from a specified root vertex to some other vertex.

Van der Hofstad, Hooghiemstra and Van Mieghem proved that the expected total length of the SPT is equal to

$$
1+\frac{1}{4}+\frac{1}{9}+\cdots+\frac{1}{n^{2}}
$$

in other words, the same number that occurs in the Parisi formula. Since the number of edges in the SPT is $n$, it follows that the average length of an edge in the SPT is given by (6).

We now notice that each edge in the SPT is the first edge of the shortest path from exactly one vertex to the root. Hence if we choose a vertex $v_{i}$ uniformly among $v_{1}, \ldots, v_{n}$ and consider the first edge of the shortest path from $v_{i}$ to $v_{0}$, then we are actually choosing an edge of the SPT with uniform distribution on its $n$ edges. Hence the expected cost of this edge is given by (6).

\section{Interpretation of the shortest path problem as an assignment problem}

It was noted by Chandra Nair (personal communication 2005) that the shortest path problem on the complete graph on $n+1$ vertices can be encoded as an assignment problem in an $n$ by $n$ matrix with entries $a(i, j)$ as follows. We label the rows by the numbers $0, \ldots, n-1$ and the columns by the numbers $1, \ldots, n$. We now simply let the matrix entries be equal to the lengths of the edges between the corresponding vertices. The entries $a(i, i)$ for $i=1, \ldots, n-1$ are all zero, but notice that with our numbering, these entries are one step off the main diagonal. It is now easily verified that an assignment in this matrix must contain a set of entries corresponding to a path from $v_{0}$ to $v_{n}$ in the graph, and conversely any such path can be completed to an assignment by combining it with the matrix entries $a(i, i)$ for the vertices $i$ that do not belong to the path. Hence the shortest path problem on the complete graph is equivalent to a special case of the assignment problem.

In the random model, the matrix entries apart from the $(n-1)$-diagonal of zeros are exponential variables of rate 1 . We have the symmetry $a(i, j)=a(j, i)$ for $1 \leq i, j \leq n-1$, but otherwise the variables are independent. It can now be seen easily in the percolation model that this symmetry is immaterial, and that the problem would essentially be the same even if each pair of vertices were connected by two directed 
edges of independent lengths. This is because in the percolation model, only the lengths of the edges from an infected to a non-infected vertex are considered. If the distances were asymmetric, so that the distance from $v_{i}$ to $v_{j}$ could be different from the distance from $v_{j}$ to $v_{i}$, then since one of the vertices must be infected before the other, only one of the two distances will ever be considered. Another way to state this is to say that there is an algorithm for finding the minimum assignment in the corresponding matrix that never reads both of $a(i, j)$ and $a(j, i)$ for $i \neq j$. Hence it does not matter whether these variables are actually equal, or just have the same random distribution.

This shows that the random shortest path problem on the complete graph $K_{n+1}$ is equivalent to the random $n$ by $n$ assignment problem with a given zero cost $(n-1)$-diagonal. We remark that assignment problems with a given set of zero cost entries have been studied by several authors [2; 3; 7]. In particular, D. Coppersmith and G. Sorkin [3] proved in 1999 that the expected value of the assignment problem with an $(n-1)$-diagonal of zeros is asymptotically $\log n / n$, which is equivalent to the corresponding result for the shortest path problem established by Janson [6] in the same year.

\section{A conjecture}

We finally mention a very natural conjecture, which can possibly be proved by the methods of [11]. As was explained in the previous section, the shortest path problem is equivalent to an assignment problem with an $(n-1)$-diagonal of zero entries in the matrix.

If a row in the matrix contains independent $\exp (1)$ entries, and we condition on the location of the minimum entry in the row, then since every minimum assignment contains exactly one entry in the row, we get an equivalent problem by subtracting the value of the minimum from each entry in the row. This leaves a zero in the position of the minimum, and by well-known properties of the exponential distribution, the remaining entries will still be independent and $\exp (1)$-distributed.

Conditioning on an $(n-1)$-diagonal of zero entries is therefore equivalent to conditioning on the minimum entries in each of these $n-1$ rows occurring in different columns. It is therefore natural to make the following conjecture:

Conjecture 1 Theorem 2 holds even if we condition on the location of the minimum entry in each of the $n-1$ rows of the matrix, or equivalently if we choose in an arbitrary way one position in each row and condition on these entries being zero.

We have seen that Theorem 2 of Nair, Prabhakar and Sharma leads to the solution of the recursive distributional equation (5) considered by Aldous. It is highly desirable to obtain a better understanding of this theorem, since its generalizations could eventually lead to the solution of other related problems, for instance the recursive distributional equation associated with the random traveling salesman problem, described in [1].

\section{References}

[1] Aldous, D., The $\zeta(2)$ limit in the random assignment problem, Random Structures \& Algorithms 18 (2001), no 4. 381-418.

[2] Alm, S. E. and Sorkin, G. B., Exact expectations and distributions in the random assignment problem, Combin. Probab. Comput. 11 (2002), no. 3, 217-248.

[3] Coppersmith, D. and Sorkin, G. B., Constructive Bounds and Exact Expectations For the Random Assignment Problem, Random Structures \& Algorithms 15 (1999), 133-144.

[4] Van der Hofstad, R., Hooghiemstra, G. and Van Mieghem, P., Size and Weight of Shortest Path Trees with Exponential Link Weights, to appear in Combinatorics, Probability and Computing.

[5] Van der Hofstad, R., Hooghiemstra, G. and Van Mieghem, P., The Weight of the Shortest Path Tree, to appear in Random Structures \& Algorithms.

[6] Janson, S., One, two and three times $\log n / n$ for paths in a complete graph with random weights, Combin. Probab. Comput. 8 (1999), 347-361.

[7] Linusson, S. and Wästlund, J., A proof of Parisi's conjecture on the random assignment problem, Probab. Theory Relat. Fields 128 (2004), 419-440.

[8] Mézard, M. and Parisi, G., Replicas and optimization, J. Phys. Lett. 46(1985), 771-778. 
[9] Mézard, M. and Parisi, G., Mean-field equations for the matching and the travelling salesman problems, Europhys. Lett. 2 (1986) 913-918.

[10] Mézard, M. and Parisi, G., On the solution of the random link matching problems, J. Phys. Lett. 48 (1987), 1451-1459.

[11] Nair, C., Prabhakar, B. and Sharma, M., Proofs of the Parisi and Coppersmith-Sorkin conjectures for the finite random assignment problem, Proceedings of IEEE FOCS, 2003.

[12] Olin, B., Asymptotic properties of the random assignment problem, Ph.D. thesis, Kungl Tekniska Högskolan, Stockholm, Sweden, (1992).

[13] Parisi, G., A conjecture on random bipartite matching, arXiv:cond-mat/9801176, 1998.

[14] Walkup, D. W., On the expected value of a random assignment problem, SIAM J. Comput., 8 (1979), 440-442. 